\title{
Improvement of Quantum Efficiency in Transmission-Type Spin-Polarized Photocathode
}

\author{
Xiuguang Jin ${ }^{1, ~ *, ~ F u m i a k i ~ I c h i h a s h i ², ~ A t s u s h i ~ M a n o ~}{ }^{3}$, Masahiko Suzuki ${ }^{4}$, Tsuneo Yasue ${ }^{4}$, \\ Takanori Koshikawa ${ }^{4}$, Yoshikazu Takeda ${ }^{5}$ \\ ${ }^{1}$ Institute for Advanced Research, Nagoya University, Furo-cho, Chikusa-ku, Nagoya, Japan \\ ${ }^{2}$ Graduate School of Engineering, Nagoya University, Furo-cho, Chikusa-ku, Nagoya, Japan \\ ${ }^{3}$ Synchrotron Radiation Research Center, Nagoya University, Furo-cho, Chikusa-ku, Nagoya, Japan \\ ${ }^{4}$ Fundamental Electronics Research Institute, Osaka Electro-Communication University, Neyagawa, Osaka, Japan \\ ${ }^{5}$ Aichi Synchrotron Radiation Center, Seto, Japan
}

Email address:

jinxg@post.kek.jp (Xiuguang Jin)

*Corresponding author

\section{To cite this article:}

Xiuguang Jin, Fumiaki Ichihashi, Atsushi Mano, Masahiko Suzuki, Tsuneo Yasue, Takanori Koshikawa, Yoshikazu Takeda. Improvement of Quantum Efficiency in Transmission-Type Spin-Polarized Photocathode. International Journal of Materials Science and Applications.

Vol. 5, No. 4, 2016, pp. 178-182. doi: 10.11648/j.ijmsa.20160504.11

Received: May 17, 2016; Accepted: August 4, 2016; Published: August 8, 2016

\begin{abstract}
We successfully developed a new transmission-type GaAs/GaAsP strained superlattice photocathode with an AlGaAs transparent inter-layer and $\mathrm{Si}_{3} \mathrm{~N}_{4}$ anti-reflection coating. The electrons emitted from this photocathode showed a high spin polarization of $90 \%$ with a quantum efficiency as high as $0.4 \%$. In the application for spin-polarized low energy electron microscope, a high emission current of over $1 \mu \mathrm{A}$ was observed at $3.6 \mathrm{~mW}$ pump laser power. Transmission electron microscopy observation revealed that there were a small disorder and some dislocations in the $\mathrm{GaAs} / \mathrm{GaAsP}$ superlattice layers. The disordered superlattice layers result in a fluctuation of the superlattice band structure and the dislocations trap the photo-electrons during the diffusion to the surface. Both of the defects influenced the performance of spin-polarized photocathode.
\end{abstract}

Keywords: Spin Polarization, Photocathode, Superlattice, Microscopy

\section{Introduction}

Highly spin-polarized electron sources based on the GaAs-type semiconductor photocathode have been developed mainly for accelerators in the field of nuclear and particles physics. A new project, i.e. an electron-ion collider, in which an intense spin-polarized electron beam collides with intense beam of both polarized nucleons and unpolarized nuclei to reveal their inner secrets. Several American institutes have been stated to construct this facility $[1,2]$. In addition, as a future plan, an international linear collider project is under considering in the government of Japan [3, 4].

Recently, these spin-polarized electron sources also have attracted considerable attention for several types of electron microscopy e.g. spin-polarized low energy electron microscopy (SPLEEM) and spin pulse transmission electron microscopy [5-8]. The spin-polarized electron beam is used as incident beam and magnetic images are obtained by electrons diffracted from a specimen. SPLEEM has superior features for the investigation of local magnetic structures in details; (1) magnetic structures are observed with a high lateral resolution of the order of $10 \mathrm{~nm}$. (2) Magnetization direction of the specimen can be determined three dimensionally without rotating and/or moving the specimen. (3) A LEEM image is obtained simultaneously in the same field of view as the magnetic image. (4) A low energy electron diffraction (LEED) pattern can be observed in a selected area in the field of view.

A strained superlattice (SL) structure composed of GaAs related semiconductors is an excellent candidate photocathode for a highly spin-polarized electron source [9, 10]. In the GaAs-type semiconductor, the spin orientations of electrons excited by circularly polarized photons from the heavy-hole 
and light-hole bands are opposite to each other. A high spin polarization was achieved by the resolution of degeneracy between the heavy-hole and light-hole bands at the $\Gamma$ point and by exciting electrons only from one band. In the strained SL structure, the heavy-hole and light-hole mini-bands are split due to both the elastic strain effect and the quantum confinement effect. The reflection-type photocathodes based on a GaAs/GaAsP strained SL [11] and an AlInGaAs/AlGaAs strained SL [12] produced a high spin polarization (SP) of approximately $90 \%$.

In our previous study, we developed the transmission-type GaAs/GaAsP strained SL photocathode [13, 14]. In this system, a super-high brightness electron beam was achieved by illuminating a small area on the backside of the photocathode with a pump laser light that was well-focused by a short-focal-length lens. To set up this system, a GaAs/GaAsP strained SL was fabricated on a GaP (bandgap energy $\left.\left(\mathrm{E}_{\mathrm{g}}\right): 2.26 \mathrm{eV}\right)$ substrate, which is transparent to the pump laser light (laser light energy (hv): 1.44 1.77 eV), instead of a GaAs $\left(\mathrm{E}_{\mathrm{g}}: 1.42 \mathrm{eV}\right)$ substrate. As a result, a super-high brightness $\left(1.3 \times 10^{7} \mathrm{~A} \cdot \mathrm{cm}^{-2} \cdot \mathrm{sr}^{-1}\right)$ and a high SP $(90 \%)$ electron beam were reproducibly achieved with this transmission-type photocathode.

The next aim for the transmission-type photocathode is the improvement of the quantum efficiency (QE). QE is defined as a ratio of the number of photo-emitted electrons to the number of incident photons. In the previous photocathode, the pump laser light irradiates the backside of the photocathode and about $30 \%$ of the pump laser light is reflected at the $\mathrm{GaP}$ surface. In addition to this reflection, about $60 \%$ of the pump laser light (hv: $1.59 \mathrm{eV}$ ) is absorbed by a 600-nm-thick GaAs inter-layer that was deposited on the GaP substrate. The GaAs inter-layer was necessary to obtain the periodic GaAs/GaAsP SL layers $[15,16]$. Therefore, about only $28 \%$ of the pump laser light reaches the 12 pairs of GaAs/GaAsP strained SL structure. Then, only several percent of the pump laser light is absorbed by the SL structure. As a result, the QE is as low as $0.1 \%$. The one of the methods to increase the $\mathrm{QE}$ is to eliminate the loss of the pump laser light before reaching the SL structure.

In this study, we successfully developed a new transmission-type GaAs/GaAsP strained SL photocathode with a $\mathrm{Si}_{3} \mathrm{~N}_{4}$ anti-reflection coating and an AlGaAs transparent inter-layer. As a result, a high spin polarization of $90 \%$ with high QE of $0.4 \%$ was achieved. The value of the QE is 4 times higher than that of the previous transmission-type photocathodes [13]. To find a clue to improve the QE further, we investigated the SL structure using the transmission electron microscopy (TEM). There still remains a small disorder and some dislocations in the SL layers. The effects of the disordered SL layers and dislocations on the photocathode performance are discussed. In the application for the SPLEEM, a high emission current of over $1 \mu \mathrm{A}$ was observed at $3.6 \mathrm{~mW}$ pump laser power. The emission current from the new photocathode is much higher than that from our previous photocathodes at same laser power.

\section{Experimental}

The refractive index $\left(\mathrm{n}_{0}\right)$ and thickness $(\mathrm{d})$ of the anti-reflection coating at the wavelength $(\lambda)$ are calculated by the following equations:

$$
\begin{aligned}
\mathrm{n}_{0} & =\sqrt{\mathrm{n}_{1} \mathrm{n}_{2}} \\
\mathrm{~d} & =\lambda / 4 \mathrm{n}_{0},
\end{aligned}
$$

where $\mathrm{n}_{1}$ and $\mathrm{n}_{2}$ are refractive indices of the first and second materials, respectively. In our case, the first and second materials are vacuum and $\mathrm{GaP}$. The refractive indices of the vacuum and $\mathrm{GaP}$ are 1 and 3.45, respectively. Equation (1) shows that the optimum refractive index is 1.86 . Therefore, $\mathrm{Si}_{3} \mathrm{~N}_{4}$, whose refractive index is 2 , was used for the anti-reflection material. In the $\mathrm{GaAs} / \mathrm{GaAsP}$ strained SL photocathode, the maximum SP was observed at a pump laser energy near $1.59 \mathrm{eV}$ (wavelength $(\lambda): 780 \mathrm{~nm}$ ). According to equation (2), the thickness of $\mathrm{Si}_{3} \mathrm{~N}_{4}$ was decided to be $100 \mathrm{~nm}$. In order to avoid absorption in the inter-layer, $\mathrm{Al}_{\mathrm{x}} \mathrm{Ga}_{1-\mathrm{x}} \mathrm{As}$ $(\mathrm{x}=0.4)$ was used instead of GaAs that was necessary to induce tensile strain in the GaAsP buffer layer [11]. $\mathrm{Al}_{0.4} \mathrm{Ga}_{0.6} \mathrm{As}\left(\mathrm{E}_{\mathrm{g}}\right.$ : $1.9 \mathrm{eV})$ is transparent to the pump laser light (hv: $1.59 \mathrm{eV})$ and the lattice constant of AlGaAs is almost the same as that of GaAs.

The photocathode structure was grown on Zn-doped (001) GaP substrates $\left(1.34 \times 10^{18} \mathrm{~cm}^{-3}\right)$ using a low-pressure organometallic vapor phase epitaxy system with a vertical cold-wall quartz reactor. The reactor pressure was fixed at 76 Torr. Triethylgallium, trimethylaluminum, tertiarybutylphosphine and tertiarybutylarsine were used as source materials. Following the growth of a $600-\mathrm{nm}$ $\mathrm{Al}_{0.4} \mathrm{Ga}_{0.6} \mathrm{As}$ inter-layer and a $1-\mu \mathrm{m} \mathrm{GaAs} \mathrm{s}_{0.65} \mathrm{P}_{0.35}$ buffer layer, 12 pairs of 4-nm GaAs and 4-nm $\mathrm{GaAs}_{0.65} \mathrm{P}_{0.35}$ strained SL layers were grown at $660^{\circ} \mathrm{C}$ with a $\mathrm{Zn}$ dopant concentration of $1.5 \times 10^{18} \mathrm{~cm}^{-3}$. Subsequently, the SL structure was coated with a highly doped 5-nm GaAs layer with a $\mathrm{Zn}$ doping of $6 \times 10^{19} \mathrm{~cm}^{-3}$. After the crystal growth, the structure was coated with a $100-\mathrm{nm}$ of $\mathrm{Si}_{3} \mathrm{~N}_{4}$ layer by Electron Cyclotron Resonance sputtering. For comparing the performance with the present photocathode, the previous photocathode with a GaAs inter-layer was also prepared.

To characterize structural details, X-ray diffraction measurements and TEM measurements were performed. The compositions of the GaAsP buffer layer and the barrier layers in the SL as well as the periods of the superlattice were determined from the peak positions. To estimate the bandgap of the superlattice structures, photoluminescence measurements were carried out at room temperature. $\mathrm{Ar}^{+}$laser light $(\lambda: 514.5 \mathrm{~nm})$ was used to excite carriers. Thus, the structural and optical characteristics of the photocathode activity layers were determined. The periods of present and previous SL are $8 \mathrm{~nm}$ and $8.6 \mathrm{~nm}$, and the band gaps of present and previous SL are $1.580 \mathrm{eV}$ and $1.566 \mathrm{eV}$, respectively. The results are summarized in Table 1. 
Table 1. Structural details of photocathodes.

\begin{tabular}{llll}
\hline Sample & P composition of GaAsP buffer layer and GaAsP barrier layer & Period of SL (nm) & SL band gap (eV) \\
\hline $\begin{array}{l}\text { SL with GaAs inter layer } \\
\text { SL with AlGaAs inter layer and } \\
\text { anti-reflection coating }\end{array}$ & 0.35 & 8.6 & 1.566 \\
\hline
\end{tabular}

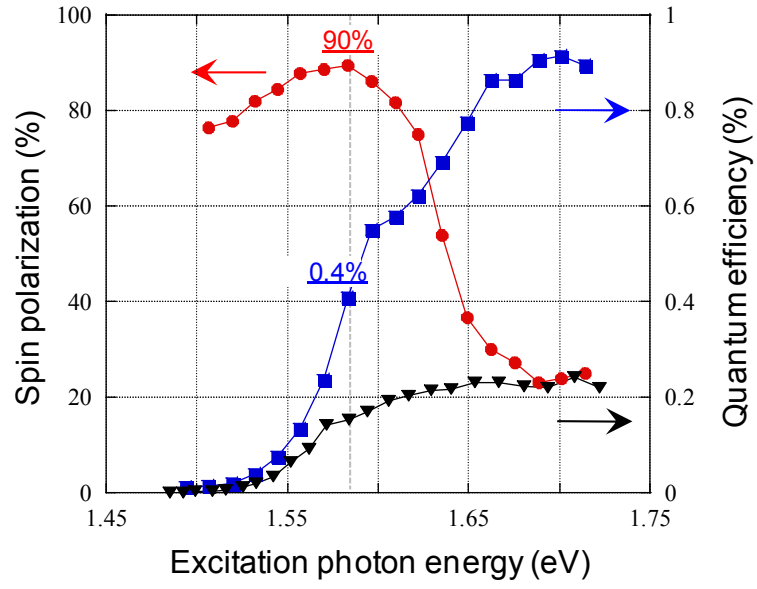

Figure 1. SP and $Q E$ as a function of excitation photon energy in present and previous photocathodes. The data points for the SP and QE spectra from the

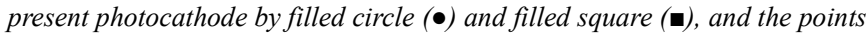
for the QE from the previous photocathode are shown by filled triangle ( $\mathbf{\nabla})$ In present photocathode, high SP of $90 \%$ with high QE of $0.4 \%$ was achieved.

The SP and the QE were measured by JPES1 [14] at room temperature. After depositing Cs and oxygen and forming a negative electron affinity (NEA) surface, the photocathode was transferred from the activation chamber to the gun chamber. The SP and QE were measured under the back side illumination arrangement of the pump laser light in the gun chamber at an extraction voltage of $20 \mathrm{kV}$.

TEM observations were performed at an electron energy of $200 \mathrm{keV}$ using a Hitachi H-800 electron microscopy. Cross-sectional TEM specimens were prepared by standard sample preparation techniques as follows: (1) two small pieces of the sample oriented along the $\left[\begin{array}{lll}1 & 1 & 0\end{array}\right]$ and $\left[\begin{array}{lll}1 & \overline{1} & 0\end{array}\right]$ directions were adhered to each other; (2) the specimens were thinned from both sides to a thickness of $20 \mu \mathrm{m}$ using a planar grinder and then polished with $\mathrm{Al}_{2} \mathrm{O}_{3}$ suspension; (3) $\mathrm{Ar}^{+}$-ion milling was carried out at $3.3 \mathrm{keV}$ under an angle $4^{\circ}$ with an ion current of $10 \mu \mathrm{A}$ until a small perforation appeared in the center of the sample.

\section{Results and Discussion}

\subsection{Energy Dependence of SP and $Q E$}

Figure 1 shows the SP and QE as a function of the photon energy of the pump laser light from the present and previous photocathodes, respectively. In the present photocathode, the QE increases with the photon energy, and the spectrum shows a distinct step at a photon energy of $1.6 \mathrm{eV}$, as expected from the density of states for SL structure. The first increase in QE corresponds to heavy-hole mini-band to conduction mini-band excitation, and the second increase corresponds to light-hole mini band to conduction mini-band excitation. The SP reaches its maximum at a slightly lower energy than the step of the QE curve. A maximum SP of $90 \%$ with a QE $0.4 \%$ was achieved. The QE values from present photocathode are much higher than that from the previous photocathode due to the elimination of the laser light loss. One more advantage about the elimination of laser light loss, the QE spectrum could provide much more information about density states of SL band.

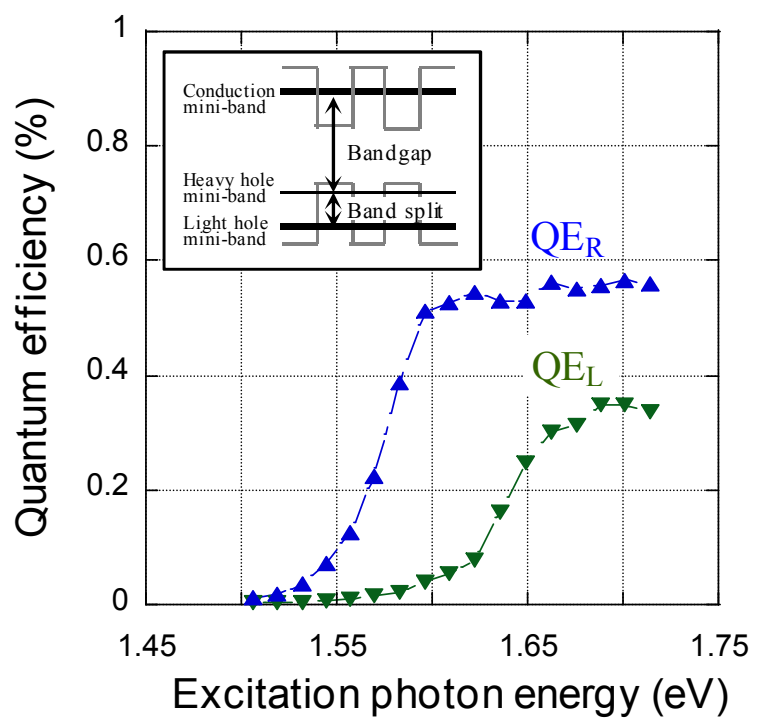

Figure 2. Spin-resolved $Q E s$ for right-handed $\left(Q E_{R}\right)$ and left-handed $\left(Q E_{L}\right)$ electrons as a function of excitation photon energy. The $Q E_{R}$ and $Q E_{L}$ spectra are excitation energy dependences of the $Q E$ from heavy-hole and light-hole mini-band to the conduction mini-band, respectively.

In the present photocathode, the SP shows its maximum slightly below the step of the QE curve. If the QE curve shows a flat region as the energy dependence of the density states of a two-dimensional structure and the maximum SP locates in that flat region, the $\mathrm{QE}$ should increase much more. In order to investigate the effect of the band structure on the SP and QE, the spin-resolved $\mathrm{QE}$ for right-handed $\left(\mathrm{QE}_{\mathrm{R}}\right)$ and left-handed $\left(\mathrm{QE}_{\mathrm{L}}\right)$ electrons were investigated. The $\mathrm{QE}_{\mathrm{R}}$ and $\mathrm{QE}_{\mathrm{L}}$ spectra are related to the experimental $\mathrm{SP}$ and $\mathrm{QE}$ spectra by $\mathrm{QE}_{\mathrm{R}}=\mathrm{QE} \times(1+\mathrm{SP}) / 2$ and $\mathrm{QE}_{\mathrm{L}}=\mathrm{QE} \times(1-\mathrm{SP}) / 2$, where the sign of the $\mathrm{SP}$ is defined as positive for right-handed electrons. The $\mathrm{QE}_{\mathrm{R}}$ and $\mathrm{QE}_{\mathrm{L}}$ spectra compared with the band structure of the $\mathrm{SL}$ are shown in figure 2. In this case, the $\mathrm{QE}_{\mathrm{R}}$ and $\mathrm{QE}_{\mathrm{L}}$ spectra are excitation energy dependences of the $\mathrm{QE}$ of electrons from the heavy-hole mini-band to the conduction mini-band, and light-hole mini-band to the conduction mini-band, respectively. When two curves are added, it gives the QE curve shown in figure 1. Ideally, $\mathrm{QE}_{\mathrm{R}}$ and $\mathrm{QE}_{\mathrm{L}}$ should increase steeply with photon energy and become flat due to the density states of the $\mathrm{SL}$ as mentioned above. The curves of $\mathrm{QE}_{\mathrm{R}}$ and $\mathrm{QE}_{\mathrm{L}}$ should to 
be $100 \mathrm{meV}$ apart from each other due to the valence band split. However, the $\mathrm{QE}_{\mathrm{L}}$ has obvious tail in the lower energy range (in the $\mathrm{QE}_{\mathrm{R}}$ flat range). This tail means a mixing of the left-handed electrons into the right-handed electrons in the flat $\mathrm{QE}_{\mathrm{R}}$ range, which started at photon energy $1.60 \mathrm{eV}$. The rate of the mixing determined the SP and it is the reason that the maximum SP is observed at the energy slightly lower than $1.60 \mathrm{eV}$.

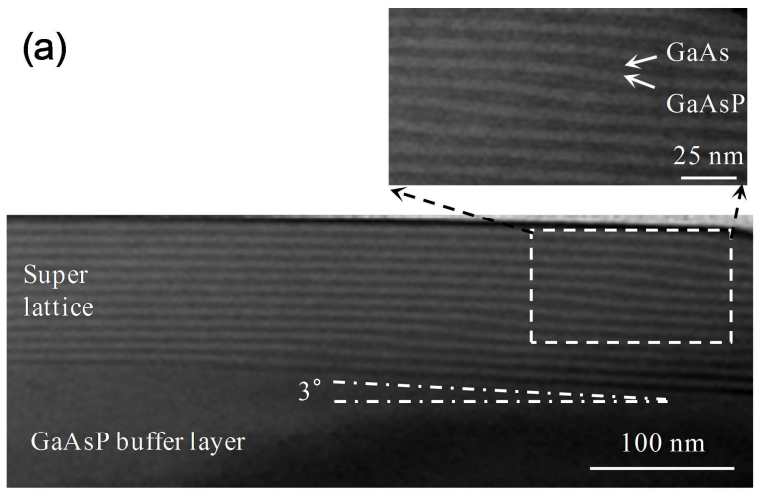

(b)

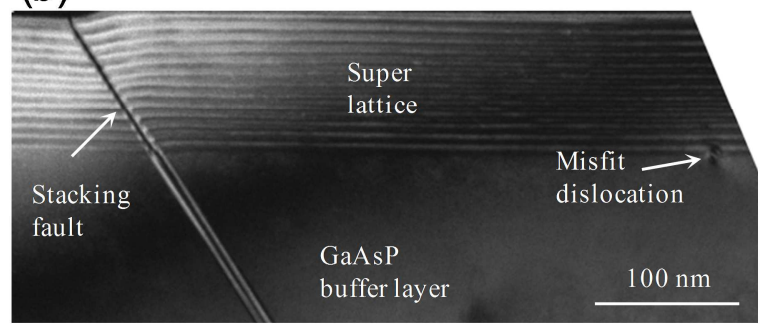

Figure 3. Cross-sectional TEM image of the superlattice on GaP substrate with AlGaAs inter-layer. The disordered superlattice layers (a) in small parts of the area and some dislocations (b) were observed.

\subsection{Structural Investigation}

The cross-sectional TEM images of the GaAs/GaAsP SL on $\mathrm{GaP}$ substrate with AlGaAs inter-layer are shown in figure 3. In the majority area, the thicknesses of the SL layers are uniform and periodic, while thicknesses of the SL on a vicinal surface are slightly disordered (figure 3 (a)). In the disordered areas, the thicknesses of the GaAs well layers are in a range of $4 \sim 6.5 \mathrm{~nm}$. The energy structures of the both SLs in periodic and disordered areas were calculated using Model-solid theory [17] and Kronig-Penny model [18]. As a result, the difference of valence band split and band gap in both areas are $5 \mathrm{meV}$ and $37 \mathrm{meV}$, respectively. With the increase of the GaAs well layer, the band split between the heavy and light hole mini-band does not change significantly. The band split keeps the large value even in the disordered areas. It indicates that the disordered SL layers do not affect the maximum SP. On the other hand, the bandgap which is the energy range between the heavy hole mini-band to the conduction mini-band, largely decreased with increase of the GaAs layer thickness. The difference of the bandgap between the periodic and disordered areas is as large as $37 \mathrm{meV}$. The SP is maximized by adjusting the excitation photon energy to the energy band gap. The oppositely spin-polarized electrons started to be excited in the disordered areas before the intended spin-polarized electrons were excited enough in the periodic area. This effect caused the tails in the $\mathrm{QE}_{\mathrm{L}}$ spectra shown in Fig. 2. Therefore, the disordered SL layers lower $\mathrm{QE}$ at the maximum SP.

Except for the disordered SL layers, some dislocations were also found as shown in figure 3 (b). Stacking fault extended from the GaAsP buffer layer into the SL layers and misfit dislocation located at the interface between GaAsP buffer layer and SL. These dislocations introduce electronic levels into band gap and trap the photo-electrons with resultant lower QE.

For realization of the transmission-type photocathode, the GaP substrate was introduced $[13,14]$. The mismatch of the lattice constant between $\mathrm{GaP}$ and $\mathrm{GaAs}_{0.65} \mathrm{P}_{0.35}$ is as large as $2.4 \%$, so it was difficult to obtain a smooth GaAsP buffer layer on the GaP substrate. The introduction of the AlGaAs (or $\mathrm{GaAs}$ ) inter-layer on the GaP substrate before the GaAsP growth successfully suppressed the roughness of the GaAsP buffer layer. However, about 25-nm height roughness was still observed. The rough GaAsP buffer layer resulted in the disordered SL layers [19, 20]. Furthermore, the GaAsP buffer layer grown on the AlGaAs inter-layer relaxed with introduction of many types of dislocations. In this case, the stacking faults dramatically influence superlattice layers because they could pass through the whole SL layers. Regarding the misfit dislocation, it caused by the strain relaxation of on the strained superlattice layers. To make the GaAsP layer flat and decrease the dislocations, the strain relaxation process and a graded buffer layers will be investigated in a future study.

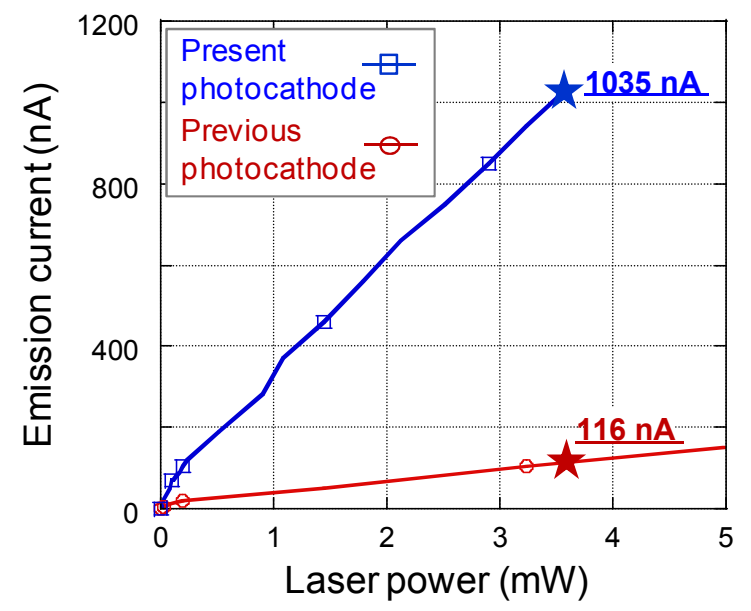

Figure 4. Emission current of electron beam as a function of the pump laser power. In present photocathode, a high current of $1035 \mathrm{nA}$ was extracted at $3.6 \mathrm{~mW}$ laser power.

\subsection{Performance of SPLEEM}

The present photocathode was installed in a SPLEEM with an extreme high vacuum gun chamber [21]. After depositing Cs and oxygen and making the NEA surface, the electron beam was extracted from the photocathode excited at a photon energy of $1.58 \mathrm{eV}$. The extracting voltage of the electron gun was $20 \mathrm{kV}$. Figure 4 shows the emission current of the electron beam as a function of the pump laser power in the present and previous photocathodes. The emission current increases 
linearly with increase of laser power in the both photocathodes In the present photocathode, a high current of $1035 \mathrm{nA}$ was extracted at $3.6 \mathrm{~mW}$ whose power was directly measured at the exit of the optical fiber. This value is about 9 times higher than that of the previous photocathode at the same laser power, which is much larger than the expected value (fourfold from the photocathode). At present the reason for the difference is not clear. The NEA surface made in the present and the previous photocathodes may be different. The electron transportation efficiency in the SPLEEM system may also be different since optimization of the transportation path was not made. However, such a big improvement can give us a big advantage because we can use much lower power of the laser device. It would also give technical advantage if one expects to make time resolved experiments to observe the fast movement of magnetic domains with such SPLEEM, which is necessary high emission current.

\section{Conclusion}

A new transmission-type photocathode with AlGaAs inter-layer and a $\mathrm{Si}_{3} \mathrm{~N}_{4}$ anti-reflection coating was developed. The QE at the maximum SP (90\%) was as high as $0.4 \%$. From the TEM investigation, the disordered SL layers were observed in small parts of area. The disordered SL layers should make fluctuation of the band structure. Therefore, the SP reached its maximum before QE increased enough to the flat region. It means the elimination of the disordered SL layers could increase QE further. In addition, some dislocations were observed in the suepralttice layers and they also lowered the QE In the application for SPLEEM, a high current over $1 \mu \mathrm{A}$ was successfully extracted from the present photocathode.

\section{Acknowledgements}

This work was supported by the Technology Development Program for Advanced Measurement and Analysis of Japan Science and Technology (JST). This work was also supported by the Grants-in-Aid for Scientific Research (A) (23246003) and Grants-in-Aid for Scientific Research (C) (25390066) from the Japan Society for the Promotion of Science (JSPS). The authors also wish to thank Professors S. Zaima and Y. Shimura of the Department of Crystal Materials Science at Nagoya University for providing advice regarding preparation of the TEM specimens.

\section{References}

[1] F. Lin, A. Bogacz, P. Brindza, Proceeding of IPAC2015 (2015) 1302.

[2] Y. Jing, V. N. Litvinenko, D. Trbojevic, Proceeding of IPAC2015 (2015) 757.

[3] S. Komamiya, Proceeding of IPAC2016 (2016) 1.

[4] B. List, L. Hagge, N. J. Walker, Proceeding of IPAC2016 (2016) 51 .
[5] M. Kuwahara, S. Kusunoki, Y. Nambo, K. Saitoh, X. G. Jin, T. Ujihara, H. Asano, Y. Takeda, N. Tanaka, Appl. Phys. Lett., 105 (2014) 193101.

[6] K. Yoshida, K. Murakami, J. Fujita, J. Vac. Sci. Technol. B32 (2014) 06FC02.

[7] O. Romanyuk, S. F. Garrido, P. Jiricek, I. Bartos, L. Geelhaar, O. Brandt, T. Taskova, Appl. Phys. Lett. 106 (2015) 021602.

[8] D. Yu, C. Math, M. Neier, M. Escher, G. Rangelov, M. Donath, Surf. Sci. 601 (2007) 5803.

[9] T. Omori, Y. Kurihara, Y. Takeuchi, M. Yoshioka, T. Nakanishi, S. Okumi, M. Tsubata, M. Tawada, K. Togawa, Y. Tanimoto, C. Takahashi, T. Baba, M. Mizuta, Jpn. J. Appl. PHys. 33 (1994) 5676.

[10] K. Togawa, T. Nakanishi, T. Baba, F. Furuta, H. Horinaka, Y. Kurihara, H. Matsumoto, T. Matsuyama, T. Nishitani, S. Okumi, T. Omori, C. Suzuki, Y. Takeuchi, K. Wada, M. Yamamoto, M. Yoshioka, Nucl. Instrum. Methods Phys. Res. A 455 (2000) 118.

[11] T. Nishitani, T. Nakanishi, M. Yamamoto, S. Okumi, F. Furuta, M. Miyamoto, M. Kuwahara, N. Yamamoto, and K. Naniwa, H. Horinaka. T. Matsuyama, K. Togawa, T. Saka, M. Tawada, T. Omori, Y. Kurihara, M. Yoshioka, K. Kato, and T. Baba, J. Appl. Phys. 97 (2005) 094907.

[12] Yu. A. Mamaev, L. G. Gerchikov, Yu. P. Yashin, D. A. Vasiliev, V. V. Kuzmichev, V. M. Ustinov, A. E. Zhukov, V. S. Mikhrin, A. P. Vasiliev, Appl. Phys. Lett. 93 (2008) 81114.

[13] N. Yamamoto, T. Nakanishi, A. Mano, Y. Nakagawa, S. Okumi, M. Yamamoto, T. Konomi, X. G. Jin, T. Ujihara, Y. Takeda, T. Ohshima, T. Saka, T. Kato, H. Horinaka, T. Yasue, T. Koshikawa, M. Kuwahara, J. Appl. Phys. 103 (2008) 064905.

[14] X. G. Jin, N. Yamamoto, Y. Nakagawa, A. Mano, T. Kato, M. Tanioku, T. Ujihara, Y. Takeda, S. Okumi, M. Yamamoto, T. Nakanishi, T. Saka, H. Horinaka, T. Kato, T. Yasue, T. Koshikawa, Appl. Phys. Express 1 (2008) 045002.

[15] X. G. Jin, Y. Maeda, T. Saka, M. Tanioku, S. Fuchi, T. Ujihara, Y. Takeda, N. Yamamoto, Y. Nakagawa, A. Mano, S. Okumi, M. Yamamoto, T. Nakanishi, H. Horinaka, T. Kato, T. Yasue, and T. Koshikawa, J. Cryst. Growth 310 (2008) 5039.

[16] T. Saka, Y. Ishida, M. Kanda, X. G. Jin, Y. Maeda, S. Fuchi, Y. Takeda, T. Matsuyama, H. Horinaka, T. Kato, N. Yamamoto, A. Mano, Y. Nakagawa, M. Kuwahara, S. Okumi, T. Nakanishi, M. Yamamoto, T. Ohshima, T. Kohashi, M. Suzuki, M. Hashimoto, T. Yasue, T. Koshikawa, e-J. Surf. Sci. Nanotech. 8 (2010) 125.

[17] Chris G. Van de Walle, Phys. Rev. B39 (1989) 1871.

[18] S. Gasiorowicz, in Quantum Physics, Chapter 5, Ed. C. Mills, (John Wiley \& Sons, Inc., New York, 1996).

[19] X. G. Jin, H. Nakahara, K. Saitoh, T. Saka, T. Ujihara, N. Tanaka, Y. Takeda, J. Cryst. Growth 353 (2012) 84.

[20] H. M. Cox, D. E. Aspnes, S. J. Allen, P. Bastos, D. M. Hwang, S. Mahajan, M. A. Shahid, P. C. Morais, Appl. Phys. Lett. 57 (1990) 611.

[21] M. Suzuki, M. Hashimoto, T. Yasue, T. Koshikawa, Y. Nakagawa, T. Konomi, A. Mano, N. Yamamoto, M. Kuwahara, M. Yamamoto, S. Okumi, T. Nakanishi, X. G. Jin, T. Ujihara, Y. Takeda, T. Kohashi, T. Oshima, T. Saka, T. Kato, H. Horinaka, Appl. Phys. Express 3 (2010) 026601. 